\title{
Immediate Implant Placement in Infected Sockets: A Consecutive Cohort Study
}

\author{
Ashish Kakar ${ }^{1,2,3^{*}}$, Kanupriya Kakar ${ }^{4}$, Minas D. Leventis ${ }^{5}$, Gaurav Jain ${ }^{6}$ \\ ${ }^{1}$ Sr. Consultant Indraprastha Apollo Hospitals and Private Practice, New Delhi, India \\ ${ }^{2}$ Adjunct Assistant Professor- Rutgers School of Dental Medicine, Newark, NJ, USA \\ ${ }^{3}$ Visiting Professor, Yenepoya University, Mangalore, India \\ ${ }^{4}$ Private Practice, New Delhi, India \\ ${ }^{5}$ Department of Oral \& Maxillofacial Surgery, Dental School, University of Athens, Athens, Greece \\ ${ }^{6}$ Clinical Associate, Dental Surgery, Indraprastha Apollo Hospitals and Private Practice, New Delhi, India
}

\section{*Correspondence to Ashish Kakar Tel: +91-9811081052; Fax: +911126823629 Email: \\ kakar_ashish@yahoo.com}

Published online March 15 2020

\begin{abstract}
Introduction: Immediate placement of implants in a fresh post-extraction socket is an increasingly popular and established treatment option. However, active infection in the extraction site may adversely affect the outcome of this procedure. This study was designed to assess the clinical results of immediate placement of dental implants in infected extraction sockets using a standardized protocol, which included (a) the use of an Er,Cr:YSGG laser for the decontamination of the infected socket prior to implant insertion, and (b) the utilization of an in situ hardening alloplastic bone graft substitute to augment the gap between the implant surface and the labial plate of bone.

Patients and Methods: A retrospective record review was used to identify 68 patients who had implants placed as per the described protocol. A total of 126 implants were placed in 68 patients (65 implants in the maxilla, 61 implants in the mandible). The implants were loaded $136 \pm 73$ days (mean \pm standard deviation; range: $37-400$ days) after implant placement. Eight patients (16 implants) were subsequently lost to follow up.

Results: 105 of the 110 implants $(95.45 \%)$ placed immediately in the infected sites using the described protocol survived after prosthetic loading.

Conclusion: Immediate implant placement in previously infected sites using the protocols mentioned in our study with laser decontamination of the socket, grafting with an in situ hardening alloplastic bone graft material and non-submerged healing shows a similar survival rate to the published success rates for immediate implants placed in non-infected sites.

Keywords: Immediate implants; Infected tooth sockets; Lasers; Bone grafting, Er-YAG laser.
\end{abstract}

\section{Introduction}

Placement of dental implants into fresh extraction sockets has major advantages over delayed placement, such as reduced overall treatment time and the number of surgical procedures. ${ }^{1}$ Animal and human studies have reported successful outcomes with immediate placement of implants, and immediate implant placement has become a routine treatment in dental practice..$^{2-5}$ The presence of active infection in the extraction site has been considered one of the main contraindications to postextraction immediate implant insertion due to the risk of spread of the infection to the peri-implant tissues, leading to implant failure or retrograde peri-implantitis. ${ }^{1}$

However, recent findings suggest that immediate implant insertion into infected sockets is not associated with inferior implant survival rates or increased risks, as compared to non-infected sockets. It has gained some acceptance as a valid treatment option to more conservative two-staged approaches. Waasdorp et $\mathrm{al}^{6}$ and Corbella et $\mathrm{al}^{7}$ evaluated implant placement in infected extraction sockets by reviewing the relevant literature. The human studies identified by Corbella et $\mathrm{al}^{7}$ reported survival rates ranging between 92 and $100 \%$ for a total of 497 implants placed in sites with endodontic infections with follow-ups of 12 months or longer. Meltzer ${ }^{8}$ placed 77 implants in infected sockets in 63 patients and reported a survival rate of $98.7 \%$ during a follow-up period of 3 to 24 months. Fugazzotto ${ }^{9}$ described a retrospective case series with 418 immediate implants placed in sites with peri-apical pathology. The implants were followed-up for 67.3 months on average, and the survival rate was $97.8 \%$.

It is evident that thorough debridement and 
decontamination of the hard and soft tissue aspects of infected sockets as well as the removal of microbial debris are prerequisites for successful immediate implant placement. The literature reports the prescription of systemic antibiotics as a prophylactic measure to reduce the infection risks. ${ }^{7}$ Antiseptic rinsing (e.g. with nonalcoholic chlorhexidine solutions) may be applied to inactivate bacteria that reside in anatomically complex spaces that are not accessible to mechanical cleaning.

Recently, the use of LASERs has become increasingly popular in dental implantology. Lasers may be used for the debridement of extraction sockets for immediate implant placement. ${ }^{10}$ On the basis of a case series which included a microbial analysis of the sockets, Kusek ${ }^{11}$ suggested an Er,Cr:YSGG laser for the decontamination of infected sockets prior to implant insertion. The Er,Cr:YSGG laser operates in combination with a water spray, using a wavelength in the infrared spectrum $(\lambda=$ $2.78 \mu \mathrm{m})$. In their review, Chrcanovic et $\mathrm{al}^{12}$ pointed out that laser treatment indeed is an interesting option to debride infected sockets prior to implantation due to the noticeable reduction of bacteria reported by Kusek, ${ }^{11}$ but the number of patients $(n=10)$ reported in the literature is low. In our dental office, laser debridement is a standard measure prior to implant placement. The present study was carried out to validate the established treatment procedure for immediate implant insertion in infected sockets in a larger series of patients.

\section{Patients and Methods}

The patient files of consecutive cases with implantation in infected extraction sites undertaken at the Global Health Research Group, New Delhi, were assessed for this retrospective analysis by the attending Dental surgeon. All surgical procedures were performed by a single surgeon (AK). The prosthodontics procedures were completed by KK. The reasons for tooth extraction were classified, as suggested by Corbella et $\mathrm{al}^{7}{ }^{7}$ for each prospective implant site as an endodontic cause, a periodontal cause, a root fracture, and a combined endodontic-periodontal cause or trauma with subsequent infection.

\section{Surgical Protocol}

Informed consent for extraction and immediate dental implant insertion was obtained from all patients. All patients started on pre-operative antibiotics 1-2 days prior to the surgical procedure (Augmentin-GlaxoSmithKline, India, $1000 \mathrm{mg}$ twice daily, in the case of penicillin allergy, a combination of Azithromycin-Alembic, India $500 \mathrm{mg}$ once daily and Ofloxacin-Cipla, India $200 \mathrm{mg}$ twice daily).

The affected teeth were extracted atraumatically (Figure 1) using minimally invasive techniques with periotomes (GDC, India) and luxators (SDI, Sweden). The extractions were performed without raising flaps. Inflammatory tissues, pus, and necrotic tissues were removed by thorough socket curettage. Care was taken to clean both the

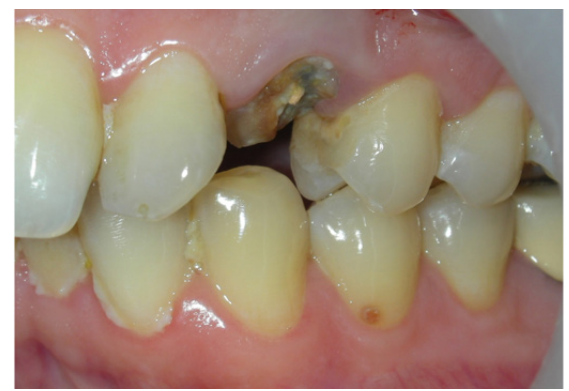

Figure 1. Preoperative Intraoral View.

hard and soft tissue aspects of the socket. The extraction sockets were considered for immediate implant insertion if the buccal plate was intact or minimally affected (e.g. perforation due to a draining sinus). Socket grafting was contemplated in cases with heavily affected buccal plates. Immediate implant insertion was not performed in such cases. After thorough mechanical cleaning, the sockets were rinsed with $5 \mathrm{ml}$ of an aqueous $0.2 \%$ chlorhexidine digluconate solution (Clohex, Dr. Reddy's Laboratories Ltd., Hyderabad, India), followed by a $5 \mathrm{~mL}$ sterile saline rinse to remove tissue debris from the socket. This procedure was repeated three times for each socket. The laser treatment was adapted and modified from the protocol described by Kusek ${ }^{11}$ and was performed before the preparation of the implant bed. An Er,Cr:YSGG laser Unit (Waterlase MD, Biolase Technology, Irvine, CA) was used for the preparation and decontamination of the socket using the MZ-4 $(14 \mathrm{~mm})$ radial-firing tip. The power was set at 1.0 to $2.5 \mathrm{~W}$ with $30 \%-40 \%$ water and $50 \%-60 \%$ air at $20 \mathrm{~Hz}$ using the Hard tissue (H-Mode) on the unit. The radial-firing tip was inserted up to the apex of the socket and slowly moved coronally, guiding the tip in a circular fashion. Application time was 40 to 90 seconds per socket, dwelling at the sites where the sources of infection were identified during tooth removal. Care was taken to debride each socket individually for multirooted teeth. The laser protocol is shown in Box 1 .

Depending on the socket morphology and location, implant design and diameter were chosen in order to

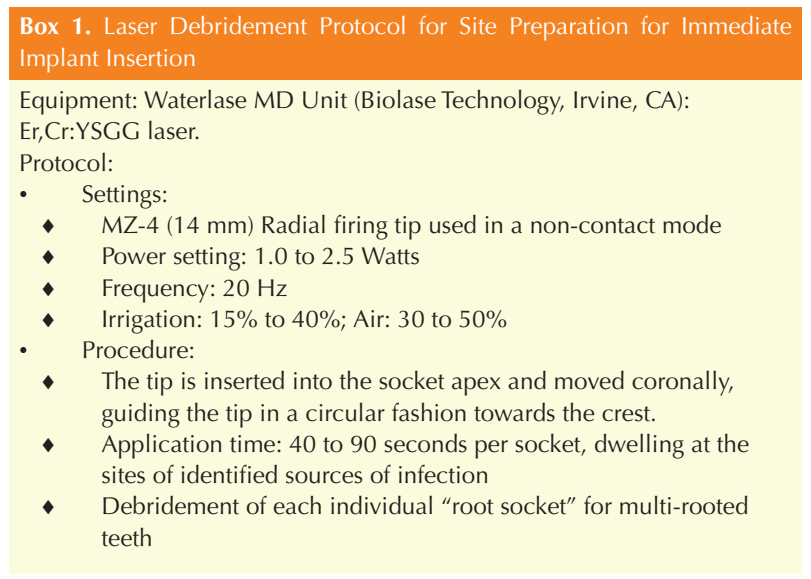


attain primary stability. Tapered implants (Nobel Active and Nobel Replace- Nobel Biocare AB, Goteborg, Sweden; Xive- Dentsply Implants, Mannheim, Germany; Alpha Bio-Petach Tikva, Israel) were used to accommodate the root morphology. For single restorations, interradicular septae were used in sockets from multi-radicular teeth to get a favorable mesiodistal implant position. For multirooted teeth which were part of a bridge, either the mesial or distal socket was chosen as the implant site. For singlerooted teeth, the socket was used as the implant site, often placing the implant in a palatal axis, especially in upper anterior teeth, for better esthetics and emergence. Where possible, implants were placed 1 to $1.5 \mathrm{~mm}$ sub-crestally (Figure 2A) to optimize the platform switch features of the implant system.

Gaps between the implant and the bony socket wall as well as any bone deficiencies caused by the pre-existing inflammatory processes were filled with an alloplastic, an in situ hardening bone graft substitute (GUIDOR easygraft CRYSTAL, Sunstar Suisse SA, Etoy, Switzerland) (Figure 2B). The moldable material was applied directly from the applicator into the defect. It was tightly packed, and the bone graft substitute surfaces were smoothened using gauze moistened with sterile saline. The material was filled up to bone level, covering the cover screw in most cases. The bone graft substitute used has the inherent ability to harden in contact with blood, thereby avoiding the need for a membrane for graft retention. The defect was sutured using a 4-0 Vicryl suture (NW2304, Ethicon, Johnson \& Johnson Ltd., India). Primary closure was not always achieved since flap elevation was kept to a minimum. In cases where primary closure was possible, the wound edges were approximated by sutures.

The patients were prescribed Ibuprofen $400 \mathrm{mg}$ (Cipla, India) and were instructed to take the medication when needed only. Antibiotics were continued for 5 to 7 days post-operatively. Mouth rinses with Chlorhexidine (Clohex Plus- Dr. Reddy's Laboratories Ltd, Hyderabad, India) solution were prescribed twice daily as an additional oral hygiene measure for the next 8 to 10 weeks. The patients were advised to refrain from brushing the surgical area for the first day after surgery. A soft surgical brush
(Surgical brush, TePe, Malmö, Sweden) was dispensed for the cleaning of the surgical area after the initial healing phase during the first 2 weeks post-surgically.

The implants were restored using the conventional protocols of delayed loading after 3 months in the mandible and after 4 months in the maxilla. They were exposed using minimal flaps or Diode lasers (Biolase Technology, Irvine, CA, USA). Standard impressions and prostheses were fabricated as per the patients' treatment plan, which ranged from single-tooth restoration to multiple teeth bridges. All prosthetic restorations were fixed, either cemented or screw-retained (Figure 3B).

\section{Results}

Sixty-eight consecutive patients (40 males, 28 females) were treated using the described protocol. The patients were $57.5 \pm 16$ years of age (mean \pm standard deviation; range: $22-85$ years) at the time point of extraction. The reasons for extraction were endodontic causes in 32 sites, periodontal causes in 18 sites, root fractures at 26 sites, combined endodontic-periodontal causes at 42 sites, and trauma with subsequent infection in 2 sites of implant placement. In 4 patients (5 Implant sites), there was a postoperative infection in the Implant area. These implants were removed and areas were re-treated with the laser protocol as described earlier and were re-grafted using an alloplastic, an in-situ hardening bone graft substitute (easy-graft Crystal, Sunstar Suisse SA, Etoy, Switzerland). These 5 sites were later re-implanted successfully after 6-8 months and restored thereafter. The data from these
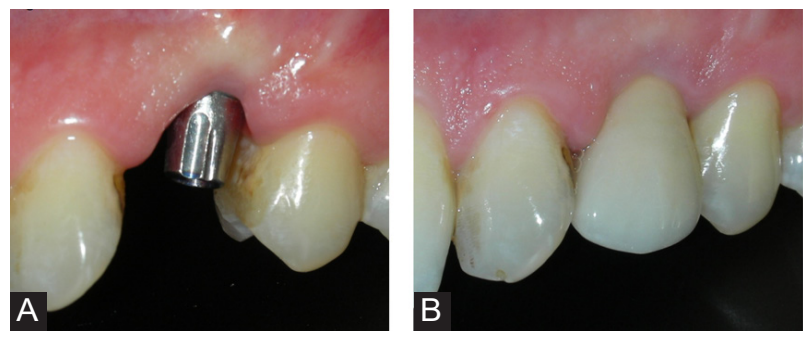

Figure 3. (A) Healed implant with the abutment installed: intraoral view. (B) Final appearance.
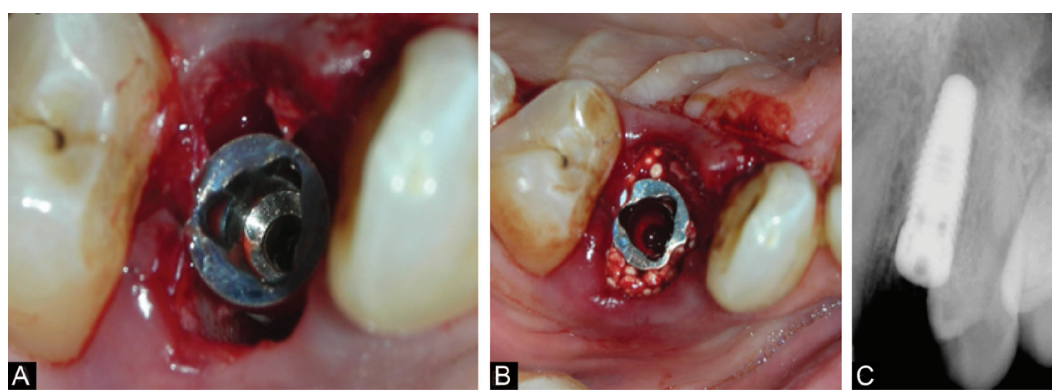

Figure 2. (A) Immediate implant inserted. (B) Jumping space grafted with an alloplastic, an in situ hardening bone graft substitute (GUIDOR easy-graft CRYSTAL, Sunstar Suisse SA, Etoy, Switzerland). (C) Post implant insertion IOPA. 
patients were recorded as failures for the purpose of this study.

In all, the 68 patients received 126 implants (65 implants placed in the maxilla and 61 in the mandible). Details are shown in Table 1.

Healing was uneventful in all patients, with minimal pain and discomfort. In cases where no primary closure was attained, patients reported some bone graft substitute particles in their mouth in the days following surgery.

Eight of 68 patients (16 implants) were lost during follow-up. These patients were followed up by the referring dentists, often overseas, and thus were not available for a recall. Out of the remaining 60 patients ( 35 males, 25 females; 110 implants), 105 implants (95.45\%) survived after the prosthetic restoration was delivered, at their last follow-up. The prosthetic restoration was delivered $4.6 \pm$ 2.4 months (mean \pm standard deviation; range: 37 to 400 days) after implant insertion. Three implants in the molar region of the mandible and two implants in the front region in the maxilla ( 1 each in a canine and premolar) failed during follow-up. One of the failed implants was removed due to an infection 2.5 months after implant placement, three implants did not show osseointegration at the reopening, and one implant was lost 4 months after loading (Table 1). 110 of the 126 implants (60 out of the 68 patients) were followed up for more than 3 months post-finalization and were included for the analysis. Mean follow-up was $48.75 \pm 13.75$ months (mean \pm standard deviation; range: 116-2184 days). One implant was lost during follow-up; that is, after loading (see above), none of the other implants failed or showed ongoing pathological processes.

\section{Discussion}

We have described a surgical technique and protocol for immediate implant insertion in infected sockets using the LASER decontamination of the infected extraction socket and an in situ hardening bone graft substitute for grafting of peri-implant defects. Sixty-eight consecutive patients who received 126 implants were treated using this method. Of these, 110 implants placed in 60 patients could be followed up for more than 3 months after final loading and were finally included in the study. The implant survival rate among these 60 patients (110 implants) was $95.45 \%$ during a mean follow-up of $48.75 \pm 13.75$ months (range: 116-2184 days)
The literature reports success rates of $92 \%-100 \%$ for immediate implantation in infected sockets, which is comparable to the $98.4 \%$ survival rate found for immediate implants in non-infected sockets. ${ }^{2,7,9,13-15}$ Several studies directly compared infected sockets with unaffected sites. A retrospective analysis assessed implantation in sockets where the teeth showed radiographic evidence of a periapical lesion in comparison with implantation into non-infected sites. ${ }^{16}$ The survival rates were 98.1 and $98.2 \%$ for implants placed in sites with periapical pathology and implants placed in sites without periapical pathology respectively; the difference was not significant. In yet another multicenter retrospective study, Zuffetti et $\mathrm{al}^{17}$ compared immediate implant placement in noninfected ( $n=334$ implants) and chronically infected ( $n=193$ implants) sites and found no differences in implant survival rates. Anitua et $\mathrm{al}^{18}$ went a step further and assessed the immediate loading of implants placed into infected sites. In their retrospective analysis of 43 implants placed in 30 patients, with a mean follow-up time of 6 years, they reported an implant success rate of $94 \%$. In a similar study, no difference was detected between immediate and delayed implant placement in sites with chronic periapical infection ${ }^{19}$ or in sites with periapical radiolucency, fistula and/or suppuration compared to sites without such pathologies. ${ }^{20}$ Yet another study used piezosurgery for site preparation and osteotomy and compared implant success rates amongst non-infected sites and those with acute or chronic infections. ${ }^{21}$ They found no significant differences in the implant survival rates at 1 year and reported no failures after loading. Thus, the survival rate found in the present case series is in agreement with the range of reported survival rates of dental implants inserted in infected sockets.

Several studies have assessed the survival rates of dental implants immediately placed in infected sockets in comparison to those placed in healthy sites. There is no evidence that points towards decreased survival rates of implants placed in infected sockets compared to placement in non-infected sites. Lee et al conducted a systematic review and meta-analysis of randomized controlled trials and non-randomized clinical controlled trials and concluded that it is safe to place immediate implants in infected sockets after thorough debridement. ${ }^{22} \mathrm{~A}$ similar study by Chen et al. ${ }^{23}$ also yielded similar results. MontoyaSalazar et $\mathrm{al}^{24}$ carried out a prospective controlled study

Table 1. Distribution of Implants Placed Across Intraoral Sites

\begin{tabular}{|c|c|c|c|c|c|c|c|c|c|c|}
\hline & \multicolumn{2}{|c|}{ Incisor } & \multicolumn{2}{|c|}{ Canine } & \multicolumn{2}{|c|}{ Premolar } & \multicolumn{2}{|c|}{ Molar } & \multicolumn{2}{|c|}{ Total } \\
\hline & Number & Failed & Number & Failed & Number & Failed & Number & Failed & Number & Failed \\
\hline Maxilla & 11 & 0 & 6 & 1 & 33 & 1 & 15 & 0 & 65 & 2 \\
\hline Mandible & 8 & 0 & 8 & 0 & 16 & 0 & 29 & 3 & 61 & 3 \\
\hline Lost to follow up & 0 & 0 & 2 & 0 & 7 & 0 & 7 & 0 & 16 & 0 \\
\hline Total & 19 & 0 & 12 & 1 & 42 & 1 & 37 & 3 & 110 & 5 \\
\hline
\end{tabular}


comparing immediate implant placement in infected and non-infected sites and used a standard preparation protocol comprising debridement, curettage, irrigation with $90 \%$ hydrogen peroxide, and decontamination with the yttrium-scandium-gallium-garnet (Er,Cr:YSGG) laser, with guided bone regeneration. They found no statistically significant difference in the implant survival rate between the groups at 3 years. However, in a metaanalysis of 7 studies including 1586 implants and 25 failures, Zhao et $\mathrm{al}^{25}$ found a $116 \%$ higher risk of implant failure amongst implants placed in infected sites, as compared to those placed in non-infected sites with borderline statistical significance. They, however, found no statistical difference between the groups in terms of marginal bone loss.

In animals, immediate implant placement was assessed in sockets with induced endodontic/periradicular lesions ${ }^{26,27}$ and ligature-induced or naturally occurring

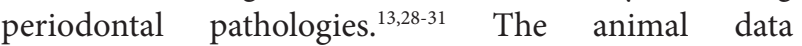
corroborated the clinical findings in terms of implant survival. Besides the general limitations of animal models, one has to be aware of issues specific to the clinical question, such as the unclear relevance of the animal infection model, and animal host reaction to remaining microorganisms. Nonetheless, only animal studies allow a histomorphometric evaluation of the bone-implant contact (BIC) and other bone-implant interactions. Two animal trials did not find any significant difference in the BIC between implants placed in infected and noninfected sites. ${ }^{26,28}$ In one study, a relevant BIC difference was detected between implants placed in sites with induced periradicular lesions compared to non-infected situations. ${ }^{27}$ Thus, based on animal experiments, it seems that previous infection may delay or reduce implant osseointegration. The implants in the animal tests were not restored. Loading of the fixtures leads to remodeling of the peri-implant bone to adapt the bony architecture to the biomechanical stimulus. ${ }^{29}$ Thus, an initial reduction of the BIC, owing to the previous infection, may not be clinically relevant due to post-loading remodeling, given that such a reduction in the BIC does not hamper implant stability. The long-term clinical success of implants placed in infected sockets argues that either functional loading indeed leads to an increase of the BIC to the same levels as for implants in non-infected sites, or that the nonsubstantial reductions in BIC, like they were observed in one of the animal studies, are without any clinical consequences. ${ }^{9,12}$

In the present case series, the gaps between the fixture and the socket walls were grafted using an in situ hardening bone graft substitute. ${ }^{32,33}$ The main reason for peri-implant grafting is to preserve the ridge contour at the implant site and to improve the marginal level of the bone-to-implant contact. ${ }^{34,35}$ Also, animal data suggest that grafting of the peri-implant gap may increase the BIC during early healing. ${ }^{30}$ Flap elevation was kept at a minimum in order to reduce the patient burden and to conserve the soft tissue anatomy. Accordingly, primary wound closure was not attained in most cases. Exposure of the bone graft substitute and non-submerged healing did not lead to complications in any of the patients, which is in accordance with the published literature. ${ }^{36,37}$ The bone graft substitute remained in the peri-implant defect due to the in situ hardening property of the material. The loss of single graft particles did not have any impact on healing in the area. The displacement of single granules from the grafted site ceased after soft tissue closure by secondary intention healing, which took 18 to 30 days, depending on the defect size.

The Er,Cr:YSGG laser is used to incise, excise and ablate intraoral soft tissues and to remove hard tissues. It has been described for the preparation of infected sockets prior to implant placement, showing that the anaerobic bacteria count was reduced by the laser treatment. ${ }^{11}$ The present case series confirmed the clinical results of the pilot study by Kusek ${ }^{11}$ in a larger patient sample, using a laser-based protocol. The main purposes of the laser treatment are to remove hard tissue that is affected by infectious and inflammatory processes and to inactivate remaining microorganisms. Furthermore, there is evidence that points towards improved healing of laser-treated tissues, ${ }^{38,39}$ which could be beneficial for bony healing in the gaps around the implant as well as for efficient soft tissue closure. However, the claims of superior healing of laser-treated sites still await confirmation in relevant, sound clinical trials.

The survival rates of implants placed in the infected socket are high after both laser-based and conventional debridement/decontamination procedures. Thus the question of the additional benefit of laser treatment is legitimate. Based on the properties of the Er,Cr:YSGG laser, it is hypothesized that laser debridement and decontamination performed in addition to mechanical cleaning may result in higher implant survival rates and less post-surgical infectious or inflammatory complications. Indeed, the results from the present case series are supportive of this hypothesis since early postsurgical complications, which would be expected as a consequence of insufficient decontamination, were not observed. However, it is beyond the scope of a case series to verify any claims; a controlled clinical trial with strict inclusion criteria and a very large patient sample will be necessary to prove or disprove a benefit of additional laser treatment. Nonetheless, the laser debridement does not pose an additional risk to the patient; thus its use for socket decontamination is justified despite the fact that the benefits have not yet been clearly shown.

\section{Conclusion}

The implant survival of $95.4 \%$ in 60 consecutive patients who received 110 immediately placed implants in previously infected sites indicates that the presented 
protocol with the laser decontamination of the socket, grafting with an in situ hardening material and nonsubmerged healing is an acceptable protocol for the management of the implant rehabilitation of patients with infected teeth requiring extraction.

\section{Ethical Considerations}

Informed consent was not required since the present study involved a retrospective analysis of patient records.

\section{Conflict of Interests}

Authors claim to have no financial interest in any company or any of the products mentioned in this article.

\section{Funding}

The authors claim to have not received funding for this work from any of the following organizations: National Institutes of Health (NIH); Welcome Trust; Howard Hughes Medical Institute (HHMI); and other(s).

\section{References}

1. Koh RU, Rudek I, Wang HL. Immediate implant placement: positives and negatives. Implant Dent. 2010;19(2):98-108. doi: 10.1097/ID.0b013e3181d47eaf.

2. Lang NP, Pun L, Lau KY, Li KY, Wong MC. A systematic review on survival and success rates of implants placed immediately into fresh extraction sockets after at least 1 year. Clin Oral Implants Res. 2012;23(Suppl 5):39-66. doi: 10.1111/j.1600-0501.2011.02372.x.

3. Smith RB, Tarnow DP. Classification of molar extraction sites for immediate dental implant placement: technical note. Int J Oral Maxillofac Implants. 2013;28(3):911-6. doi: 10.11607/jomi.2627.

4. Atalay B, Öncü B, Emes Y, Bultan Ö, Aybar B, Yalçin S. Immediate implant placement without bone grafting: a retrospective study of 110 cases with 5 years of followup. Implant Dent. 2013;22(4):360-5. doi: 10.1097/ ID.0b013e31828edd02.

5. Rodrigo D, Martin C, Sanz M. Biological complications and peri-implant clinical and radiographic changes at immediately placed dental implants. A prospective 5-year cohort study. Clin Oral Implants Res. 2012;23(10):1224-31. doi: 10.1111/j.1600-0501.2011.02294.x.

6. Waasdorp JA, Evian CI, Mandracchia M. Immediate placement of implants into infected sites: a systematic review of the literature. J Periodontol. 2010;81(6):801-8. doi: 10.1902/jop.2010.090706.

7. Corbella S, Taschieri S, Tsesis I, Del Fabbro M. Postextraction implant in sites with endodontic infection as an alternative to endodontic retreatment: a review of literature. J Oral Implantol. 2013;39(3):399-405. doi: 10.1563/AAID-JOI-D-11-00229.

8. Meltzer AM. Immediate implant placement and restoration in infected sites. Int $J$ Periodontics Restorative Dent. 2012;32(5):e169-73.

9. Fugazzotto P. A retrospective analysis of immediately placed implants in 418 sites exhibiting periapical pathology: results and clinical considerations. Int J Oral Maxillofac Implants. 2012;27(1):194-202.
10. Romanos GE, Gupta B, Yunker M, Romanos EB, Malmstrom H. Lasers use in dental implantology. Implant Dent. 2013;22(3):282-8. doi: 10.1097/ID.0b013e3182885fcc.

11. Kusek ER. Immediate implant placement into infected sites: bacterial studies of the Hydroacoustic effects of the YSGG laser. J Oral Implantol. 2011;37:205-211. doi: 10.1563/AAID-JOI-D-10-00014.

12. Chrcanovic BR, Martins MD, Wennerberg A. Immediate placement of implants into infected sites: a systematic review. Clin Implant Dent Relat Res. 2015;17(Suppl 1):e1e16 . doi: 10.1111/cid.12098.

13. Marcaccini AM, Novaes AB Jr, Souza SL, Taba M Jr, Grisi MF. Immediate placement of implants into periodontally infected sites in dogs. Part 2: A fluorescence microscopy study. Int J Oral Maxillofac Implants. 2003;18(6):812-9.

14. Jofre J, Valenzuela D, Quintana P, Asenjo-Lobos C. Protocol for immediate implant replacement of infected teeth. Implant Dent. 2012;21(4):287-94. doi: 10.1097/ ID.0b013e31825cbcf8.

15. Palmer R. Evidence for survival of implants placed into infected sites is limited. J Evid Based Dent Pract. 2012;12(3 Suppl):187-8. doi: 10.1016/S1532-3382(12)70034-9.

16. Fugazzotto PA. A retrospective analysis of implants immediately placed in sites with and without periapical pathology in sixty-four patients. J Periodontol. 2012;83(2):182-6. doi: 10.1902/jop.2011.110016.

17. Zuffetti F, Capelli M, Galli F, Del Fabbro M, Testori T. Postextraction implant placement into infected versus noninfected sites: A multicenter retrospective clinical study. Clin Implant Dent Relat Res. 2017;19(5):833-840. doi: 10.1111/cid.12523.

18. Anitua E, Piñas L, Alkhraisat MH. Long-Term outcomes of immediate implant placement into infected sockets in association with immediate loading: A retrospective cohort study. J Periodontol. 2016;87(10):1135-40. doi: 10.1902/ jop.2016.160104.

19. Lindeboom JA, Tjiook Y, Kroon FH. Immediate placement of implants in periapical infected sites: a prospective randomized study in 50 patients. Oral Surg Oral Med Oral Pathol Oral Radiol Endod. 2006;101(6):705-10. doi: 10.1016/j.tripleo.2005.08.022.

20. Siegenthaler DW, Jung RE, Holderegger C, Roos M, Hämmerle $\mathrm{CH}$. Replacement of teeth exhibiting periapical pathology by immediate implants: a prospective, controlled clinical trial. Clin Oral Implants Res. 2007;18(6):727-37. doi: 10.1111/j.1600-0501.2007.01411.x.

21. Blus C1, Szmukler-Moncler S, Khoury P, Orrù G. Immediate implants placed in infected and noninfected sites after atraumatic tooth extraction and placement with ultrasonic bone surgery. Clin Implant Dent Relat Res. 2015;17(Suppl 1):e287-97. doi: 10.1111/cid.12126.

22. Lee J, Park D, Koo KT, Seol YJ, Lee YM. Comparison of immediate implant placement in infected and noninfected extraction sockets: a systematic review and metaanalysis. Acta Odontol Scand. 2018;76(5):338-45. doi: 10.1080/00016357.2018.1453084.

23. Chen H, Zhang G, Weigl P, Gu X. Immediate placement of dental implants into infected versus noninfected sites in the esthetic zone: A systematic review and meta-analysis. J Prosthet Dent. 2018;120(5):658-667. doi: 10.1016/j. prosdent.2017.12.008. 
24. Montoya-Salazar V, Castillo-Oyagüe R, Torres-Sánchez C, Lynch CD, Gutiérrez-Pérez JL, Torres-Lagares D. Outcome of single immediate implants placed in post-extraction infected and non-infected sites, restored with cemented crowns: a 3-year prospective study. J Dent. 2014;42(6):64552. doi: 10.1016/j.jdent.2014.03.008.

25. Zhao D, Wu Y, Xu C, Zhang F. Immediate dental implant placement into infected vs. non-infected sockets: a metaanalysis. Clin Oral Implants Res. 2016;27(10):1290-1296. doi: $10.1111 /$ clr.12739.

26. Novaes AB Jr, Vidigal GM Jr, Novaes AB, Grisi MF, Polloni S, Rosa A. Immediate implants placed into infected sites: a histomorphometric study in dogs. Int J Oral Maxillofac Implants. 1998;13(3):422-7.

27. Chang SW, Shin SY, Hong JR, Yang SM, Yoo HM, Park DS, et al. Immediate implant placement into infected and noninfected extraction sockets: a pilot study. Oral Surg Oral Med Oral Pathol Oral Radiol Endod. 2009;107(2):197203. doi: 10.1016/j.tripleo.2008.06.003.

28. Novaes AB Jr, Marcaccini AM, Souza SL, Taba M Jr, Grisi MF. Immediate placement of implants into periodontally infected sites in dogs: a histomorphometric study of bone-implant contact. Int $J$ Oral Maxillofac Implants. 2003;18(3):391-8.

29. Berglundh T, Abrahamsson I, Lindhe J. Bone reactions to longstanding functional load at implants: an experimental study in dogs. J Clin Periodontol. 2005;32(9):925-32. doi: 10.1111/j.1600-051X.2005.00747.x.

30. Tehemar S, Hanes P, Sharawy M. Enhancement of osseointegration of implants placed into extraction sockets of healthy and periodontally diseased teeth by using graft material, an ePTFE membrane, or a combination. Clin Implant Dent Relat Res. 2003;5(3):193-211. doi: 10.1111/ j.1708-8208.2003.tb00202.x.

31. Papalexiou V, Novaes AB Jr, Grisi MF, Souza SS, Taba M $\mathrm{Jr}$, Kajiwara JK. Influence of implant microstructure on the dynamics of bone healing around immediate implants placed into periodontally infected sites. A confocal laser scanning microscopic study. Clin Oral Implants Res. 2004;15(1):44-53. doi: 10.1111/j.1600-0501.2004.00995.x.

32. Schmidlin PR, Nicholls F, Kruse A, Zwahlen RA, Weber FE. Evaluation of moldable, in situ hardening calcium phosphate bone graft substitutes. Clin Oral Implants Res. 2013;24(2):149-57. doi: 10.1111/j.1600-0501.2011.02315.x.

33. Jurišić M, Manojlović-Stojanoski M, Andrić M, Koković V, Danilović V, Jurišić T, et al. Histological and morphometric aspects of ridge preservation with a moldable, in situ hardening bone graft substitute. Arch Biol Sci. 2013;65(2):429-37. doi:10.2298/ABS1302429J.

34. Chen ST, Darby IB, ReynoldsEC. A prospective clinical study of non-submerged immediate implants: clinical outcomes and esthetic results. Clin Oral Implants Res. 2007;18(5):552562. doi: 10.1111/j.1600-0501.2007.01388.x.

35. Araújo MG, Linder E, Lindhe J. Bio-Oss collagen in the buccal gap at immediate implants: a 6-month study in the dog. Clin Oral Implants Res. 2011;22(1):1-8. doi: 10.1111/j.1600-0501.2010.01920.x.

36. Schwartz-Arad D, Chaushu G. Immediate implant placement: a procedure without incisions. J Periodontol. 1998;69(7):743-750. doi: 10.1902/jop.1998.69.7.743.

37. Barber HD, Lignelli J, Smith BM, Bartee BK. Using a dense PTFE membrane without primary closure to achieve bone and tissue regeneration. J Oral Maxillofac Surg. 2007;65(4):748-52. doi: 10.1016/j.joms.2006.10.042.

38. Pourzarandian A, Watanabe H, Aoki A, Ichinose S, Sasaki $\mathrm{KM}$, Nitta $\mathrm{H}$, et al. Histological and TEM examination of early stages of bone healing after Er:YAG laser irradiation. Photomed Laser Surg. 2004;22(4):342-350. doi: 10.1089/ pho.2004.22.342.

39. Khadra M. The effect of low level laser irradiation on implant-tissue interaction. In vivo and in vitro studies. Swed Dent J Suppl. 2005;172:1-63. 